\title{
Effects of Nano-selenium on Blood Biochemistry, Liver Antioxidant Activity and GPx-1 mRNA Expression in Rabbits
} \author{
Shun-yi QIN ${ }^{1}$, Fu CHEN ${ }^{2}$, Fang-hong ZHAO $^{1}$, Tian-ming $\mathrm{JIN}^{1}$ and
Ji-fei MA \\ ${ }^{1}$ College of Animal Science and Veterinary Medicine, Tianjin Agriculture University, \\ Tianjin 300384, China \\ ${ }^{2}$ College of Animal Science and Technology, Qingdao Agriculture University, Qingdao \\ 266109, China
}

*Corresponding author, email: hbmjfts@126.com

Keywords: Nano-selenium, Rabbit, Blood biochemistry, Antioxidant activity, glutathione peroxidase

\begin{abstract}
The experiment was conducted to compare the effect of nano-selenium and sodium selenite on blood biochemistry, liver antioxidant activity and GPx-1 mRNA expression in rabbit. Twenty-four 10-week-old New Zealand rabbits, female and male in half, were randomly divided into three groups. Rabbits in Group 1 were fed a basal diet as control, while rabbits in Groups 2 and 3 were fed the basal diet supplemented with $0.3 \mathrm{mg} / \mathrm{kg}$ selenium as sodium selenite or nano-selenium, respectively. The experiment lasted for 42 days. Blood biochemical values, glutathione peroxidase (GPx) activity, total antioxidant capacity (T-AOC), malondialdehyde (MDA) content and glutathione peroxidase I (GPx-1) mRNA expression in liver were determined at experiment termination. The results showed that blood biochemistry of rabbits, including concentrations of cholesterin, triglyceride, total protein, glucose, alanine amino transferase and aspartate amino transferase were not significantly affected by supplementation of nano-selenium or sodium selenite. Liver GPX and T-AOC activities in nano-selenium group and sodium selenite group were significantly higher or higher than those in control group $(P<0.01$ or $P<0.05)$, respectively. Liver MDA contents in nano-selenium group and sodium selenite group were significantly lower than those in control group $(P<0.01)$, while liver MDA contents in nano-selenium group were lower than those in sodium selenite group $(P<0.05)$. Liver mRNA expression of GPx-1 was approx $195 \%$ higher in nano-selenium group and approx $154 \%$ higher in sodium selenite group than that in control group. While, there was no significant difference in the increase of liver GPx-1 mRNA expression between sodium selenite and nano-selenium groups $(P>0.05)$. In conclusion, nano-selenium supplementation could significantly enhance liver antioxidant activity and GPx-1 mRNA expression in rabbits, it was more available than sodium selenite in increasing liver antioxidant activity in rabbits.
\end{abstract}

\section{Introduction}

Selenium, as an essential trace element for normal physiological function in animals and humans, plays an important role in growth, reproduction, antioxidative mechanism, hormone metabolism, anti-carcinogenesis and immunomodulation [1]. Chemical structures are important determinants of bioavailability of selenium compounds. The mainly nutritional selenium source used in practice is sodium selenite. Nevertheless, the narrow range between its nutritional dosage and the tolerable upper intake level 
severely compromises wide applications. Recently, selenium nanoparticle (nano-selenium) is attracting increasing attention due to its high bioavailability and low toxicity $[2,3]$. However, there was not an appreciation of blood biochemistry and liver glutathione peroxidase I (GPx-1) genes expression affected by nano-selenium in rabbit in the literature. Accordingly, the objective of this study was to determine the effects of nano-selenium on blood biochemistry, liver antioxidant activity and GPx-1 mRNA expression in rabbits.

\section{Materials and Methods}

Twenty-four 10-week-old New Zealand rabbits ((bisexual each half, Animal Center, Chinese Academy of Military Medical Sciences, Beijing), at average body weight of $1300 \mathrm{~g}$ were allotted to three groups. Each group was replicated four times with four cages of two rabbits per cage in each replicate. Rabbits in Group 1 were fed a basal diet as control, while rabbits in Groups 2 and 3 were fed the basal diet supplemented with $0.3 \mathrm{mg} / \mathrm{kg}$ selenium as sodium selenite (Sigma, St. Louis, MO) or nano-selenium (Department of Animal Science, Tianjin Agricultural University, China), respectively. The basal diet was calculated to be adequate in protein, energy, vitamins, and minerals for this class of animal, except for selenium content. The experiment lasted for 42 days. During the experiment, feed and water were provided ad libitum.

\section{Sample Collection and Processing}

Blood without anticoagulation was centrifuged at $23,000 \times g$ for 20 min at $4{ }^{\circ} \mathrm{C}$ and the sera were separated. Sera were placed in the refrigerator till the further tests were run on each of the samples. All rabbits harvested under general anaesthesia using halothane at the end of experiment. Samples of liver, which were excised from each of the individual rabbit under aseptic environment, were immediately frozen in liquid nitrogen and stored at $-80^{\circ} \mathrm{C}$ until analysis as follow.

\section{Laboratory Assay}

Measurement of Blood Biochemistry. The concentrations of cholesterin (CHO), triglyceride (TG), total protein (TP), glucose(GLU), alanine amino transferase (ALT) and aspartate amino transferase (AST) were determined using an auto analyser machine (Sysmes XT-1800I, Japan) using the appropriate commercial kits (Sysmex International Reagents Co., Ltd., Japan).

Measurement of Liver Antioxidant Activity. Glutathione peroxidase (GPx) activity, total antioxidant capacity (T-AOC) activity and malondialdehyde (MDA) content in liver were determined using the kits (Nanjing Jiancheng Bioengineering Institute, Nanjing, China) following the procedure of the manufacturer's instruction.

\section{Real-time PCR for Quantification of Liver GPx-1 Gene Expression}

RNA Extraction and Reverse Transcription. Total cellular RNA was extracted from the liver cell with TRIZOL reagent as recommended by the supplier (TaKaRa). Total RNA was dissolved in $30 \mu \mathrm{l}$ Rase-free water and stored at $-70^{\circ} \mathrm{C}$. cDNA was synthesized as recommended (TaKaRa RNA PCR Kit (AMV) Ver. 3.0).

Real-time PCR SYBR Green Detection Quantification. Oligonucleotide sequences were derived from the genes for GPx1 (GenBank accession number: X13837) and GAPDH (GenBank accession number: NM_001082253). Oligo Software (version 5.0) was used to design the primers. Sequences of primers and expected PCR products length are shown in Table 1. The PCR was performed in the Lightcycler (Roche) using 
the SYBR Green I PCR kit as recommended by the manufacturer. Each reaction contained $2.0 \mu \mathrm{L}$ of the Hotstart Fluo-PCR mix, $0.5 \mu \mathrm{L}$ of Sense primer, $0.5 \mu \mathrm{L}$ of Anti-sense primer, $1 \mu \mathrm{L}$ of a 1:10 dilution of the cDNA; and $8 \mu$ RNase-free water. Reactions were performed in capillary type reaction tube (Roche). The PCR protocol consisted of denaturation at $95^{\circ} \mathrm{C}$ for $5 \mathrm{~min}$ and then by 40 cycles of $95^{\circ} \mathrm{C}$ for $20 \mathrm{~s}$ and $60^{\circ} \mathrm{C}$ for $30 \mathrm{~s}$. Acquisition mode was single, set at the end of elongation stage. To verify that the SYBR green dye detected only one PCR product, the PCR reactions were subjected to a melting curve analysis present in LightCycler software (Roche Molecular Biochemical,version 3.5).

Real-time PCR Data Analysis. For the real-time assays, the influence of nano-selenium on GPx-1 expression was evaluated by a relative quantification method $\left(2^{-\triangle \Delta C t}\right.$ method)[4].

Table 1 Sequences of primers for real-time PCR assay

\begin{tabular}{cccc}
\hline \multirow{2}{*}{ Items } & Primers & Products \\
\cline { 2 - 4 } & Sequence & $\begin{array}{c}\text { Length } \\
(\mathrm{bp})\end{array}$ & Length (bp) \\
\hline GAPDH & F:5'- CTGAACGGGAAACTCACTGG -3' & 20 & $118 \mathrm{bp}$ \\
& R:5'- TCACCACCTTCTTGATGTCG -3' & 20 & \\
GPx-1 & F:5'-GATGAACGAGCTGCAAGAGC-3' & 20 & $130 \mathrm{bp}$ \\
& R:5'- AGGCCGGACATACTTGAGG-3' & 19 & \\
\hline
\end{tabular}

\section{Statistical Analysis}

The data were analyzed using the one-way analysis of variance, followed by multiple comparisons test (LSD). The statistical analyses were performed by SPSS statistical software, version 17.0. Data were expressed as means \pm standard deviation.

\section{Results}

\section{Blood Biochemistry}

During the experiment period, blood biochemical values of rabbits, including $\mathrm{CHO}$, TG, TP, GLU, ALT and AST were not significantly affected by supplementation of nano-selenium or sodium selenite $(P>0.05$, Table 2$)$.

Table 2 Effects of nano-selenium on blood biochemistry in rabbits

\begin{tabular}{ccccccc}
\hline & CHO & TG & GLU & TP & ALT & AST \\
\hline Group 1 & $1.17 \pm 0.24$ & $1.26 \pm 0.25$ & $8.79 \pm 0.37$ & $86.64 \pm 4.05$ & $70.81 \pm 24.60$ & $128.84 \pm 23.75$ \\
Group 2 & $1.18 \pm 0.26$ & $1.33 \pm 0.34$ & $8.94 \pm 0.41$ & $84.87 \pm 5.24$ & $73.46 \pm 21.10$ & $125.56 \pm 13.81$ \\
Group 3 & $1.23 \pm 0.24$ & $1.32 \pm 0.19$ & $8.60 \pm 0.49$ & $87.69 \pm 4.46$ & $73.46 \pm 19.76$ & $116.83 \pm 15.07$ \\
\hline
\end{tabular}

\section{Liver Antioxidant Activity}

Liver GPx activities in nano-selenium group and sodium selenite group were significantly higher or higher than those in control group $(P<0.01$ or $P<0.05$, Table 3$)$. Liver T-AOC activities in nano-selenium group and sodium selenite group were significantly higher than those in control group $(P<0.01$, Table 3$)$, respectively. Liver MDA contents in nano-selenium group and sodium selenite group were significantly lower than those in control group $(P<0.01)$, while liver MDA contents in nano-selenium group were lower than those in sodium selenite group $(P<0.05$; Table 3$)$. 
Table 3 Effect of nano-selenium on liver antioxidant Activity in rabbits

\begin{tabular}{cccc}
\hline & Group I & Group II & Group III \\
\hline GSH-Px/ (U/mg protein ) & $250.14 \pm 59.82 \mathrm{Aa}$ & $325.54 \pm 48.77 \mathrm{ABb}$ & $390.67 \pm 68.56 \mathrm{Bc}$ \\
T-AOC/ (U/mg protein ) & $0.43 \pm 0.08 \mathrm{~A}$ & $0.59 \pm 0.08 \mathrm{~B}$ & $0.80 \pm 0.07 \mathrm{C}$ \\
$\mathrm{MDA} /$ (nmol/mg protein) & $0.40 \pm 0.06 \mathrm{~B}$ & $0.31 \pm 0.06 \mathrm{Ab}$ & $0.24 \pm 0.06 \mathrm{Aa}$ \\
\hline
\end{tabular}

Note:The different superscript capital letters (lowercase letters) in the same line mean a significant difference at the $0.01(0.05)$ level.

\section{GPx-1 mRNA Expression}

The relative amount of GPx-1 mRNA at each group was quantified and showed in figure 1. Liver mRNA expression of GPx-1 was approx 195\% higher in nano-selenium group and approx $154 \%$ higher in sodium selenite group than that in control group. While, there was no significant difference in the increase of liver GPx-1 mRNA expression between sodium selenite and nano-selenium groups $(P>0.05)$.

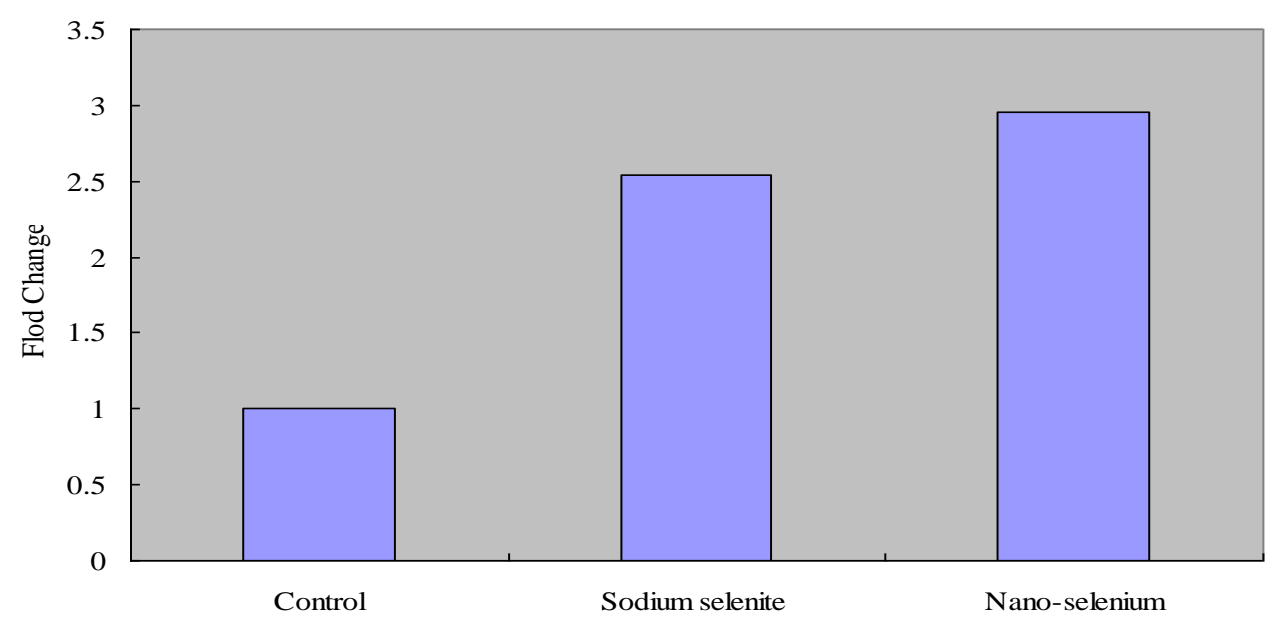

Figure 1. Effects of nano-selenium on GPx-1 mRNA expression in rabbits liver

\section{Discussion}

There was not an appreciation of blood biochemistry affected by nano-selenium in rabbit in the literature. In the present study, our results indicated that there were no effects of nano-selenium supplementation on Blood Biochemistry in rabbits, which was similar to the previous research on poultry[5].

GPx, T-AOC and MDA have been used as indicators of antioxidant activity in animals for a long time [6]. Few studies showed that nano-selenium intake could enhance the antioxidant activity of the animals [7-8]. Our results showed that both nano-selenium and sodium selenite supplementation led to the improvement of liver GPx and T-AOC activities and decrease of MDA contents in rabbits, and there were the higher liver GPx and T-AOC activities and the lower MDA contents in rabbits supplemented with nano-selenium than those supplemented with sodium selenite (Table 3), which indicated that nano-selenium could improve liver antioxidant activity in rabbits and was more effective than sodium selenite in increasing liver antioxidant activity in rabbits.

Previous studies have shown that expression of GPx-1 mRNA in tissue are highly dependent on chemical form of selenium in animals [9], However, as far as authors know, no study was undertaken on the effects of nano-selenium on expression of GPx-1 
mRNA in the liver of rabbits. Our results also showed that supplementation of nano-selenium and sodium selenite in rabbits lead to approximately $195 \%$ and $154 \%$ improvement of liver GPx-1 mRNA levels (Figure 1), respectively. And no significant difference in the increase of liver GPx-1 mRNA Expression were found when supplementation of nano-selenium or sodium selenite in rabbits. Similarly, Wang et al. reported that no significant difference were found in kindey GPx-1 mRNA expression of weanling pigs supplemented with nano-selenium or sodium selenite[10].In contrast to these results, Wang et al. also reported that nano-selenium were more effective than sodium selenite in increasing the expression level of GPx mRNA in the liver and spleen tissues of piglets[10]. Qin et al. found that nano-selenium was more bioavailable than sodium selenite in the increasing of kindey GPx-1 mRNA expression in mice[11].These results might indicate that the effects of nano-selenium on GPx-1 mRNA expression are discriminative between different tissues and different animals.

\section{Conclusions}

Nano-selenium supplementation could significantly enhance liver antioxidant activity and GPx-1 mRNA expression in rabbits. Nano-selenium was more available than sodium selenite in increasing liver antioxidant activity in rabbits.

\section{Acknowledgements}

This research was supported by Tianjin Research Program of Application Foundation and Advanced Technology (grant number: 15JCYBJC30600), Program of Veterinary Biotechnology Outstanding Scientific Research Innovation Team of Tianjin (grant number:TD12-5019) and Large Instruments Sharing Program of Tianjin Agriculture University (grant number: 2015016).

\section{References}

[1] B. R. Cardoso, B. R. Roberts, A. I. Bush, D. J. Hare, Selenium, selenoproteins and neurodegenerative diseases, Metallomics. 7 (2015) 213-1228.

[2] L. G. Shi, R. J. Yang, W. B. Yue, W. J. Xun, C. X. Zhang, Y. S. Ren, L. Shi, F. L. Lei, Effect of elemental nano-selenium on semen quality, glutathione peroxidase activity, and testis ultrastructure in male Boer goats. Anim. Reprod. Sci.;118 (2010) 248-254.

[3] B. Sarkar, S. Bhattacharjee, A. Daware, P. Tribedi, K. K. Krishnani, P. S. Minhas, Selenium nanoparticles for stress-resilient fish and livestock. Nanoscale. Res. Lett. 10 (2015) 371.

[4] T. D. Schmittgen, B. A. Zakrajsek, A. G.. Mills, V. Gorn, M. J. Singer, M. W. Reed, Quantitative reverse transcription-polymerase chain reaction to study mRNA decay: comparison of endpoint and real-time methods, Anal. Biochem. 285 (2000) 194-204.

[5] H. Ma, Effects of nano-selenium and iodine on specific egg yolk antibody production and related blood biochemical indicator in laying hen, Dissertation, Sichuan Agricultural University, China. (2007).

[6] D. Armstrong, R. Browne, The analysis of free radicals, lipid peroxides, antioxidant enzymes and compounds related to oxidative stress as applied to the clinical chemistry laboratory. Adv. Exp. Med. Biol. 366 (1994) 43-58. 
[7] Y. Wang, Differential effects of sodium selenite and nano-Se on growth performance, tissue se distribution, and glutathione peroxidase activity of avian broiler, Biol. Trace. Elem. Res. 128 (2009) 184-190.

[8] F. Zhu, L. Zhu, J. Sun, X. Meng, L. Zou, Effects of dietary supplemental nano-se levels on liver selenium content and antioxidant abilities in hens, Chinese Journal of Veterinary Science. 30 (2010) 1537-1539 In Chinese.

[9] S. L. Weiss, J. K. Evenson, K. M. Thompson, R. A. Sunde, Dietary selenium regulation of glutathione peroxidase mRNA and other selenium-dependent parameters in male rats, J. Nutr. Biochem. 8 (1997) 85-91

[10]X. Wang, Z. Geng, Z.Wu, Y. Wang, J. Zhang, Effects of Sources and Levels of Dietary Selenium on the Expression Level of mRNA of Celluar Glutathione Peroxidase Gene in Piglets, Chinese Journal of Animal Nutrition. 22 (2010) 1630-1635 In Chinese.

[11]S. Qin, F. Chen, Y. Guo, B. Huang, J. Zhang, J. Ma, Effects of nano-selenium on kindey selenium contents, glutathione peroxidase activities and GPx-1 mRNA expression in mice. Adv. Mater. Res. 1051 (2014) 383-387 